\title{
CRABP2 involvement in a mechanism of Golgi stress and tumor dry matter in non-small cell lung cancer cells via ER dependent Hippo pathway
}

\author{
Jian-Feng Meng ${ }^{1} \bowtie$ and Ming-Jie Luo ${ }^{2}$ \\ 'Department of Respiratory and Critical Care Medicine, The People's Hospital of Guangxi Zhuang Autonomous Region, Nanning, Guangxi, Chi- \\ na; 'Department of Respiratory and Critical Care Medicine, Nanxishan Hospital of Guangxi Zhuang Autonomous Region, Guilin, Guangxi, China
}

Objective: The paper aimed to explore the mechanism of cellular retinoic acid binding protein 2 (CRABP2) involvement in Golgi stress and tumor dryness in nonsmall cell lung cancer (NSCLC) cells through the estrogen receptor (ER) dependent Hippo pathway. Methods: Human NSCLC cell line A549 was purchased from ATCC andcultured in RPMI-1640 with 10\% FBS. Attractene reagent was used for plasmid transfection. ER (sh) RNA was designed using RNAi Designer. Seventy-six hours after infection, stable cells were obtained after treated with puromycin for 3 weeks. ER silencing cells (with inhibited ER expression) were compared to the control cells (normal cultured NSCLC cell line A549, CRABP2 normal expression). CRABP2 and ER expression levels were detected by RT-PCR. MTT assay was used to detect cell proliferation, and the cell localization of ER and Golgi was observed by confocal microscopy. The invasion and metastasis of cells were analyzed by Boden chamber invasion and migration assays. Western blotting assays was used for detecting the protein expression of E-cadherin, vimentin, ZO-1 protein and epithelial-mesenchymal transition (EMT) related factors. Results: The lower expression level of mRNA was detected in the ER-silencing group compared to the control group $(P<0.05)$. We also found a higher proliferation level of cells, the number of invading and metastatic cells, the expression of vimentin, p-Lats1T1079, Lats1 and p-YAPS127 mRNA in the control group compared to the ER silencing group $(P<0.05)$. And the expression level of protein kinase RNA-like endoplasmic reticulum kinase (PERK), phosphorylate eukaryotic initiation factor 2 (p-eIF2 alpha), activating transcription factor 4 (ATF4) and C/EBP-homologous protein (CHOP) in the control group was higher than that in the ER silencing group $(P<0.05)$. Adversely, a lower expression level of $E$-cadherin and ZO-1 protein was found in the control group compared to the ER silencing group $(P<0.05)$. Conclusion: The expression of CRABP2 in NSCLC cells was regulated by ER, and cell proliferation and invasion were regulated by the Hippo pathway. At the same time, it was found that decreased expression of CRABP2 enhanced endoplasmic reticulum/Golgi stress response.

Keywords: CRABP2; ER; Hippo pathway; non-small cell lung cancer cells; Golgi apparatus; tumor stem

Received: 12 November, 2020; revised: 01 February, 2021; accepted: 25 February, 2021; available on-line: 21 December, 2021

凶e-mail: mengjianfengm@sina.com

Abbreviations: ATF4, ctivating transcription factor 4; CHOP, C/ EBP-homologous protein; CRABP2, regenerated human intracellular retinoic acid binding protein-2; HCC, hepatocellular carcinoma; EMT, epithelial-mesenchymal transition; ER, estrogen receptor;
FGF, fibroblast growth factor; FGFR1, fibroblast growth factor receptor 1; NSCLC, non-small cell lung cancer; p-elF2 alpha, phosphorylate eukaryotic initiation factor 2; PERK, protein kinase RNAlike endoplasmic reticulum kinase; RA, retinoic acid; RAR, retinoic acid receptor

\section{INTRODUCTION}

Regenerated human intracellular retinoic acid binding protein-2 (CRABP2) is an intracellular lipid binding protein associated with retinoic acid, which is regarded as a key regulator of retinoic acid signal transduction in cells (Zhang et al., 2019b). CRABP2 takes part in cell proliferation, apoptosis, invasion and metastasis through transporting retinoic acid (RA) to retinoic acid receptor (RAR) in the nucleus (Feng et al., 2019, Zhu et al., 2019). Several studies indicate that CRABP2 can act as a transcription coactivator (Wei et al., 2019). In addition, CRABP2 can involve in biological behavior independent of RA or its receptor (Sung et al., 2019). Abnormal expression of CRABP2 is associated with human malignancy (Zhang et al., 2019a). Decreasing the level of CRABP2 will inhibit the movement of cancer cells and down-regulate the number of receptors on the surface of CRABP2 expression, thereby limiting cell proliferation in vitro (Wu et al., 2019). It will induce apoptosis and block cell migration (Xia et al., 2019) in esophageal squamous cell carcinoma. Previous studies have reported that estrogen receptor alpha $(\mathrm{ER} \alpha$ or ER) regulates CRABP2 transcription in some way (Liu et al., 2019). High level of CRABP2 mRNA is associated with the prognosis of patients with non-small cell lung cancer (NSCLC) (Weng et al., 2019). It is not clear whether CRABP2 is involved in regulating the invasion and metastasis of NSCLC (Chen et al., 2020). The Hippo pathway controls the development of organs by regulating cell apoptosis and cell proliferation. Recently, it has been confirmed that the Hippo pathway is closely related to the proliferation, survival, invasion and metastasis of NSCLC cells (Zhang et al., 2020). ER is a nuclear steroid receptor, expressed in approximately $75 \%$ of breast cancers. Some reports have shown that estrogen can promote the growth of NSCLC (Yang et al., 2019). However, studies have shown that the loss of ER can lead to epithelial-mesenchymal transition (EMT) and the occurrence of tumor metastasis. This study focused on exploring the mechanism of CRABP2 involvement in Golgi stress and tumor stem growth in NSCLC cells through ER dependent Hippo pathway. 


\section{MATERIALS AND METHODS}

\section{Cells and gene silencing}

The human NSCLC cell line A549 with 10\% FBS was used for cell culture, purchasing from ATCC. RPMI1640 (Gibco, USA). Attractene reagent (Qiagen, Germany) was used for plasmid transfection $(0.12 \mu \mathrm{g}$ per well in 6-well plates). ER and control siRNA were obtained from Dharmacon (GE Healthcare, USA). SiRNA (0.2 nmol/well in 6-well plates) was transfected with DharmaFECT (GE Healthcare, USA). ER (sh) RNA was designed using RNAi Designer (US Invitrogen): shER, 5'-GAATGTCAGACAGACGCGAAAAT'T-3'. pENTR/U6 was used to generate vectors encoding shRNA. Empty plasmid was used as the negative control. All cells were infected with lentivirus for sh-NC expression (sh-NC) or control lentivirus. The stable cells were obtained after 76 hours treated with puromycin for 3 weeks. Stable transfected cells were selected through culture of (Cayman Chemical, Ann Arbor, USA). The cells were selected with G418 (Sigma) at a concentration of $0.5 \mathrm{mg} / \mathrm{mL}$. In the experiment, the cells were divided into the control group (normal cultured NSCLC cell line A549, CRABP2 normal expression) and the ER silencing group (cells with inhibited ER expression).

\section{METHODS}

\section{Analysis of migration and invasion of cells}

The Boyden chamber was consisted by two compartments separated by a polycarbonate membrane. The membrane contains pores of uniform size of $8.0 \mu \mathrm{m}$. To carry out migration analysis, $200 \mu \mathrm{L}$ serum-free medium containing $2 \times 10^{5}$ cells was placed in the upper compartment, and a complete medium with $20 \%$ FBS was placed in the lower compartment. The cell chamber was removed and the extra cells were removed by gently cleaning the upper surface with a cotton swab after 12 hours of regular incubation,. The entire filter membrane was immersed in a fixed solution (4\% POM) for 30 minutes. The hematoxylin eosin staining kit (Solarbio) was used for staining. The number of stained cells was determined under a microscope at $\times 200$ magnification in 5 fields of view and the average count was calculated. The microporous filter membrane, coated with Matrigel (BD BioCoat) to form a bioactive three-dimensional matrix, was used for invasion assay. Besides the incubation time of 24 hours, the procedure was the same as the migration assay. After removing the cells above the top chamber, the cells were photographed and counted.

RNA separation and real time RT-PCR. The total RNA extract was obtained by using RNA kit I (Omega Bio-Tek, Inc., Norcross, Georgia, USA) and PrimeScript ${ }^{\text {TMRT }}$ Master Mix (Takara Biotechnology (Dalian) Co., Ltd., Liaoning, China). The cells were seeded in 12-well plates overnight at a density of $5 \times 10^{4} /$ well and treated for 24 hours with DIM (25 and $50 \mathrm{uM}$ ). The SYBR-Green I kit (M Dalian Co., Ltd., Dalian, China) was used for Real time polymerase chain reaction (RTPCR). RNAiso Plus reagent (Takara, Dalian, China) was used for Total RNA isolation. Ct was normalized to endogenous beta actin $(\Delta \mathrm{Ct}=\mathrm{Cttarget}-\mathrm{Ct} \beta$-actin) and compared with the calibrator using the $\Delta \Delta \mathrm{Ct}$ method $(\Delta \Delta \mathrm{C} \mathrm{t}=\Delta$ Ctsample- $\Delta$ Ctcontrol) for quantifying the relative expression of each gene.

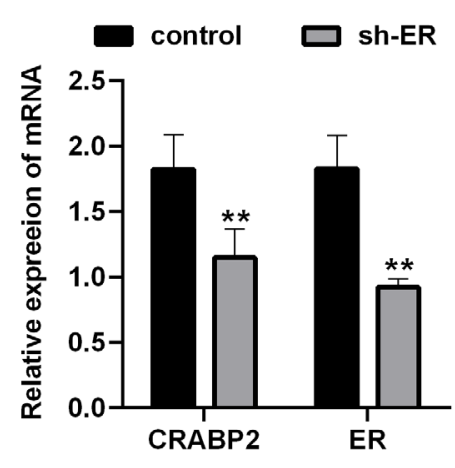

Figure 1. The mRNA expression levels of CRABP2 and ER in a control group and ER silencing group.

sh-ER, ER silencing group. ${ }^{*} P<0.05,{ }^{*} P<0.01$, ${ }^{* *} P<0.001$.

Preparation and protein blotting of cell extracts. Proteins were separated using SDS-PAGE and transferred to polyvinylidene fluoride (PVDF) (Bio-Rad, California, USA). The membranes were sealed with $5 \%$ skim milk for 2 hours. Lamin A/C and GAPDH served as controls. Cell grading analysis was carried by nuclear and cytoplasmic extraction agents (pioneer Biotech Corp, Xi'an, China). Mst2, p-Lats1T1079, Lats1, p-YAP S127, E-cadherin and ZO-1 antibodies were purchased from CST (US Cell Signaling Technology). Lamin A/C, GAP$\mathrm{DH}$, and vimentin were purchased from Proteintech, China. CRABP1, CRABP2 and anti-ER were obtained from Abcam, UK. The protein bands were visualized by image Master II scanner (GE Healthcare, Milwaukee, WI, USA). E-cadherin, vimentin, E-calcium sticky eggs, protein kinase RNA-like endoplasmic reticulum kinase (PERK), phosphorylate eukaryotic initiation factor 2 (p-eIF2 alpha), activating transcription factor 4 (ATF4) and $\mathrm{C} / \mathrm{EBP}-$ homologous protein (CHOP) antibodies were used. ImageJ Pro was used for protein quantification. Relative protein expression was standardized with the level of $\beta$-actin in each lane.

Determination of MTT. Cell proliferation was measured by MTT. Transfected $5 \times 10^{4}$ A549 cells were incubated for 96 hours at $37^{\circ} \mathrm{C}$ in 96 well plates. Cells were incubated at $37^{\circ} \mathrm{C}$ for 4 hours after $20 \mu \mathrm{L}$ of $5 \mathrm{mg} / \mathrm{mL}$ MT'T (Sigma, St. Louis, MO, USA) solution was added,. The medium was removed from each well and the resulting MTT was dissolved in $150 \mu \mathrm{L}$ DMSO. The results were quantified by spectrophotometry at $490 \mathrm{~nm}$.

Statistical analysis. All experiments were repeated at least three times in vitro. Data were analyzed using GraphPad Prism 5 (GraphPad Software, Inc., La Jolla, CA, USA). These results were displayed as mean \pm S.D. The comparison between the two groups was conducted by t test. Two-way ANOVA and Dunnett's multiple comparison test were used in two-way comparison. All statistical tests were two-sided. $P<0.05$ was considered statistically significant.

Table 1. The mRNA expression level of CRABP2 and ER in control groups and ER silencing group.

\begin{tabular}{lll}
\hline Group & CRABP2 & ER \\
\hline Control & $1.83 \pm 0.26$ & $1.83 \pm 0.25$ \\
Sh-Er & $1.15 \pm 0.22$ & $0.92 \pm 0.07$ \\
\hline
\end{tabular}



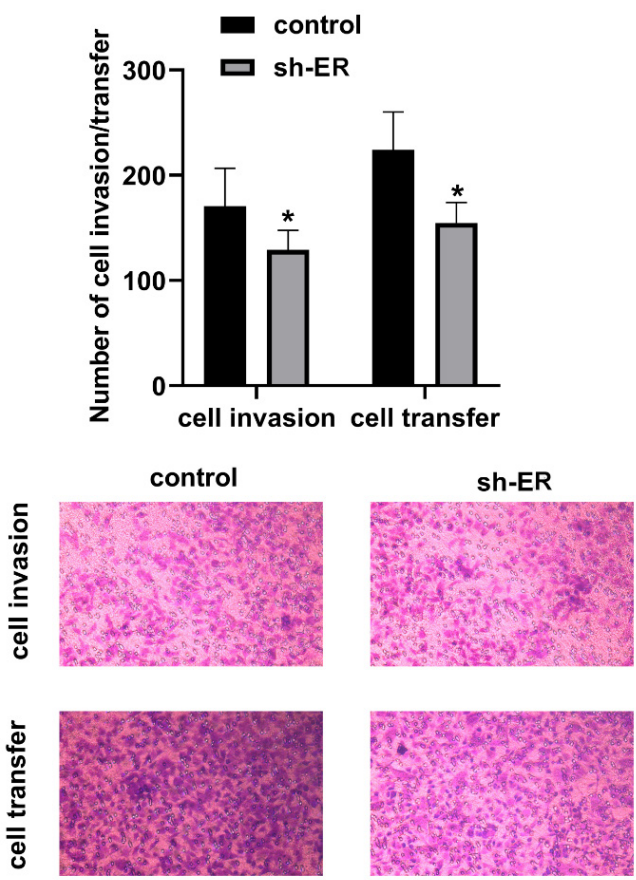

Figure 2. The outcomes of cell invasion and cell transfer in a control group and ER silencing group.

sh-ER, ER silencing group. ${ }^{*} P<0.05,{ }^{* *} P<0.01$, ${ }^{* * *} P<0.001$.

\section{RESULTS}

\section{ER regulates the mRNA expression of CRABP2}

The mRNA expression levels of CRABP2 and ER in cells were detected by RT-PCR. Compared with the control group, the mRNA expression levels of CRABP2 and ER decreased in the ER silencing group $(P<0.05)$. These results indicated that CRABP2 expression depended on ER, which promoted the expression of CRABP2 (Fig. 1, Table 1).

\section{CRABP2 promoted cell migration and invasion}

Using a lentiviral vector, the function correlation of CRABP2 gene expression restricted cells was tested. Compared with the control group, the number of invasive and metastasized cells was decreased in the ER silencing group $(P<0.05)$ (Fig. 2, Table 2$)$.

Table 2. The outcomes of cell invasion and cell transfer in control groups and ER silencing group.

\begin{tabular}{lcc}
\hline Group & Cell invasion & Cell transfer \\
\hline Control & $170.67 \pm 36$ & $224 \pm 36.00$ \\
\hline Sh-Er & $129 \pm 18.52$ & $154.67 \pm 19.50$ \\
\hline
\end{tabular}

Table 3. The outcomes of cell proliferation assay in control groups and ER silencing group.

\begin{tabular}{lll}
\hline Group & 24 hours & 48 hours \\
\hline Control & $178.08 \pm 16.87$ & $234.95 \pm 15.72$ \\
\hline sh-Er & $100.29 \pm 11.33$ & $116.70 \pm 31.77$ \\
\hline
\end{tabular}

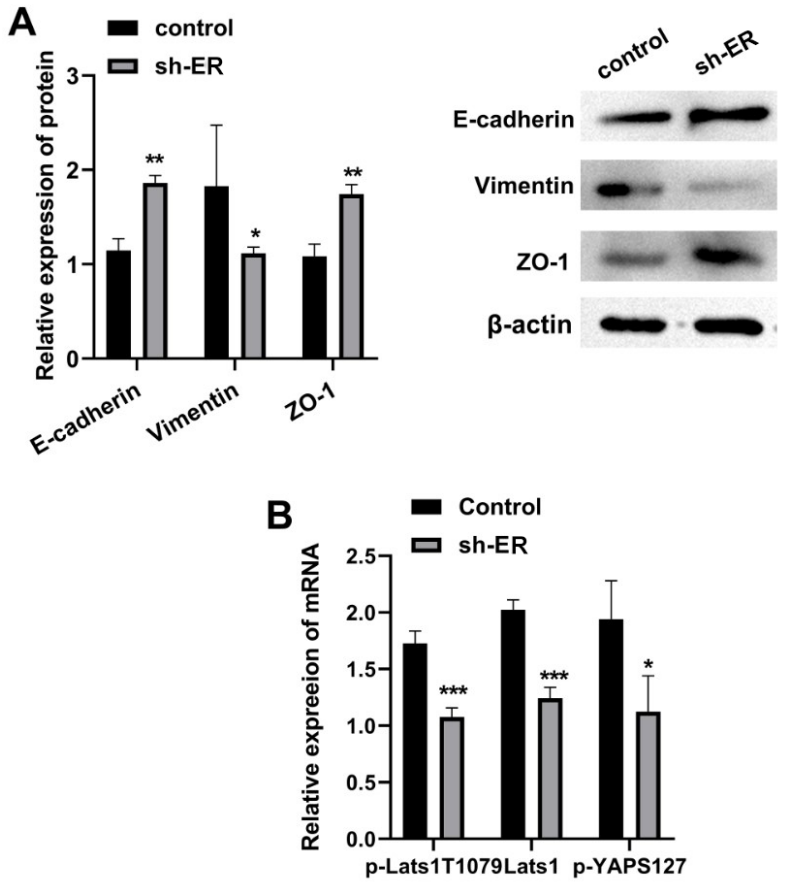

Figure 3. (A) The protein expression of $E$-cadherin, vimentin and ZO-1 in a control group and ER silencing group. (B) The mRNA expression levels of p-Lats1T1079, Lats1 and p-YAPS127 in a control group and ER silencing group.

sh-ER, ER silencing group. ${ }^{*} P<0.05,{ }^{* *} P<0.01,{ }^{* * *} P<0.001$.

\section{Cell proliferation assay}

MT'T analysis showed that the proliferation of cells cultured for 24 hours and 48 hours in the control group was significantly higher than the ER silence group $(P<0.05)$ (Supplementary Fig. 1 at https://ojs. ptbioch.edu.pl/index.php/abp/, Table 3).

\section{CRABP2 promotes cell EMT production}

The outcomes of western blot analysis showed that the expression of E-cadherin, vimentin and ZO-1 protein in the ER silencing group was higher than that in the control group $(P<0.05)$, and the expression of vimentin in the ER silencing group was lower than that in the control group $(P<0.05)$. The results indi-

Table 4. The protein expression of E-cadherin, vimentin and ZO-1 in control groups and ER silencing group

\begin{tabular}{llll}
\hline Group & E-cadherin & Vimentin & ZO-1 \\
\hline Control & $1.14 \pm 0.13$ & $1.83 \pm 0.65$ & $1.08 \pm 0.13$ \\
\hline sh-Er & $1.86 \pm 0.09$ & $1.12 \pm 0.06$ & $1.74 \pm 0.10$ \\
\hline
\end{tabular}

Table 5. The mRNA expression levels of p-Lats1T1079, Lats 1 and p-YAPS127 in control groups and ER silencing group.

\begin{tabular}{llll}
\hline Group & p-Lats1T1079 & Lats1 & p-YAPS127 \\
\hline Control & $1.73 \pm 0.12$ & $2.0 \pm 0.09$ & $1.94 \pm 0.34$ \\
\hline sh-Er & $1.1 \pm 0.08$ & $1.24 \pm 0.10$ & $1.12 \pm 0.32$ \\
\hline
\end{tabular}



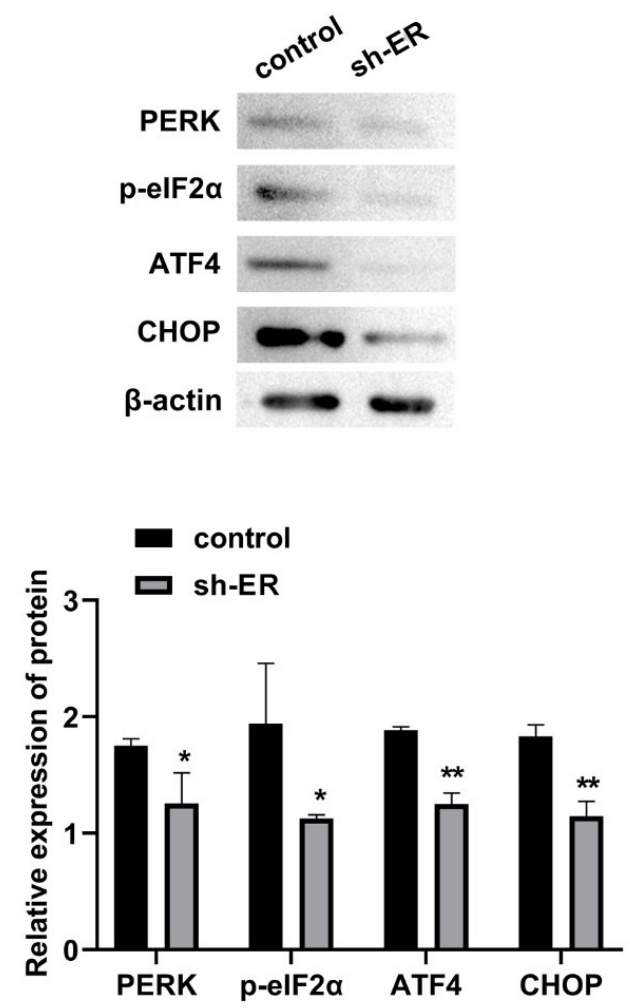

Figure 4. The protein expression of protein kinase like endoplasmic reticulum kinase (PERK), eukaryotic initiation factor 2 (p-elF2a), activated transcription factor 4 (ATF4), and C/EBP homologous protein (CHOP) in a control group and ER silencing group.

sh-ER, ER silencing group. ${ }^{*} P<0.05$, ${ }^{* *} P<0.01,{ }^{* * *} P<0.001$.

cated that CRABP2 promoted the occurrence of cell EMT (Fig. 3, Table 4).

\section{CRABP2 activates the Hippo pathway}

The mRNA expression of factors related to Hippo pathway was analyzed by real time PCR. The expression level of p-Lats1T1079, Lats1 and p-YAPS127 mRNA in the control group was higher than the ER silencing group $(P<0.05)$, indicating that CRABP2 promoted the occurrence of EMT, metastasis and invasion by activating the Hippo pathway (Table 5).

\section{Effect of CRABP2 on endoplasmic reticulum/Golgi stress} in cells

Considering the subcellular localization of endoplasmic reticulum and Golgi apparatus, we examined whether CRABP2 affected endoplasmic reticulum/Golgi apparatus stress regulatory protein. The expression levels of protein kinase like endoplasmic reticulum kinase

Table 6. The protein expression of protein kinase like endoplasmic reticulum kinase (PERK), eukaryotic initiation factor 2 (p-elF2a), activated transcription factor 4 (ATF4), and C/EBP homologous protein (CHOP) in control groups and ER silencing group.

\begin{tabular}{lllll}
\hline Group & PERK & p-elF2a & ATF4 & CHOP \\
\hline Control & $1.75 \pm 0.06$ & $1.94 \pm 0.52$ & $1.88 \pm 0.03$ & $1.83 \pm 0.10$ \\
\hline sh-Er & $1.26 \pm 0.26$ & $1.13 \pm 0.03$ & $1.25 \pm 0.10$ & $1.15 \pm 0.13$ \\
\hline
\end{tabular}

(PERK), eukaryotic initiation factor 2 (p-eIF2 $\alpha)$, activated transcription factor 4 (ATF4), and C/EBP homologous protein (CHOP) were detected by Western blotting assays. The expression levels of PERK, p-eIF2, ATF4 and CHOP in cytoplasm of the control group were higher than the ER silencing group $(P<0.05)$ (Fig. 4, Table 6).

\section{DISCUSSION}

Lung cancer is one of the leading causes of death in the worldwide. The high mortality rate of the disease could be caused by delayed diagnosis due to unobvious symptoms. According to a study, about $57 \%$ of lung cancer would metastasize and affect the normal function of other organs and tissue (Huang et al., 2019). The lung cancer is commonly divided into NSCLC and small cell lung cancer (SCLC) (Masciale et al., 2019). NSCLC is the most common type of lung cancer, accounting for $80-$ $85 \%$ of all patients. Usually, it is a slow and persistent tumor that will metastase and spread to nearby tissues and cells eventually (Guo et al., 2019). SCLC is characterized by rapid initial transmission, with more smokers than non-smokers. NSCLC and SCLC cause about 1.5 million deaths every year. However, due to the heterogeneity and metastasis of NSCLC, most studies have been carried out on NSCLC. According to statistics, only $5 \%$ of NSCLC patients can survive after stage IV. Clinical choices such as surgery, chemotherapy, radiotherapy and other cancer therapies have been used to treat such cancers, but there is still a need for better curative effects to cure cancer, especially at the advanced stage. Because of unknown symptoms, the prognosis of NSCLC is very difficult. In early stage NSCLC, most patients do not have symptoms. Therefore, diagnosing the disease at the initial stage is challenging. The formation of tumor biomarkers is very important for early diagnosis, prognosis and individualized treatment of cancer patients (including NSCLC patients) (Piao et al., 2019). In recent years, the new strategy of identifying sensitive and reliable biomarkers for accurate diagnosis and reliable prediction of recurrence has aroused great interest.

CRABP212 has two isoforms (CRABP1 and CRABP2), which have high affinity for all RA trans ligands (Cortesi \& Ventura, 2019). These same isoforms are highly conserved among species, displaying 74\% sequence identity in humans. The destruction of any CRABP subtype is highly correlated with mild limb defects (Miao et al., 2019). CRABP1 is a passive carrier that binds and releases its ligand according to the concentration gradient. CRABP2 can transmit RA to RAR and the channelization of RA between CRABP2 and RAR will increase overall RAR formation and RAR transcription (Gong et al., 2019). The anticancer mechanism of CRABP2, which does not depend on RA, has been suggested. The anticancer effect of CRABP2 has been suggested in astrocytomas, head and neck tumors. It is generally considered that CRABP2 enhancement of RA signal is an anti-tumorigenic activity. However, there are still some conflicting reports about the exact role of CRABP2 in tumorigenesis, which need to be further studied. Recent proteomic analysis found that high levels of CRABP2 are a poor prognostic marker for ERnegative breast tumors, while another study found that CRABP2 is a subtype-specific biomarker for ovarian cancer. The expression in serous ovarian cancer is upregulated, and CRABP2 expression is positively correlated with tumor grade and stage of cancer. In addition, it has 
been found that RNA-Seq in NSCLC cancer tissue is 4.9 times higher than that in normal lung tissue, and $72.1 \%$ of NSCLC samples show the expression of CRABP2 by immunohistochemistry (Han et al., 2014).

YAP is the major transductor of the Hippo pathway. It induces target genes including Connective tissue growth factor $(C T G F)$, which can induce cell proliferation and apoptosis. Previous studies have shown that Yap overexpression will result in the growth and invasion of lung cancer cells and is associated with poor survival as well (Zhao et al., 2020). The clear need for more personalized treatments has led to the development of molecularly targeted drugs. Targeted therapy is more effective than non-targeted therapy for patients with lung adenocarcinoma. As a single drug, individual targeted therapy is only clinically useful in patients with known molecular abnormalities and cancer drivers. In order to provide more effective treatment for a larger group of patients, we must identify other targeted carcinogenic pathways and/or selection criteria. A reasonable combination of such therapies that can interact with signal transduction pathways is also a potential option to improve clinical utility. The fibroblast growth factor (FGF) family consists of 18 ligands and 5 receptors, involving in cellular basic activities, including proliferation, wound healing and angiogenesis. It has been shown that aberrant signaling of this pathway promotes tumorigenesis. The amplification of fibroblast growth factor receptor 1 (FGFR1) gene in NSCLC occurred in $22 \%$ of squamous cell carcinoma and $4 \%$ of adenocarcinoma, which is related to poor overall survival and short disease-free survival (Weiss et al., 2010). Recently, an activation mutation has been found in the FGFR2 and FGFR3 genes of squamous cell lung cancer. The co-expression of FGFR and its corresponding ligands, such as FGF2 and FGF9, has been found in NSCLC, indicating the autocrine mechanism of the proliferation signaling pathway (Ware et al., 2013). In this study, the mRNA expression of Hippo pathway related factors in PCR cells was analyzed by RT-PCR. The expression levels of p-Lats1T1079, Lats1 and p-YAPS127mRNA in the control group were higher than the ER silencing group, indicating that CRABP2 promoted EMT, metastasis and invasion through activating the Hippo pathway.

The occurrence and proliferation of lung tumors is associated with ER pathway. In this study, MTT analysis showed that the proliferation of cells cultured for 24 and 48 hours in the control group was higher than the ER silencing group. A population study has shown the role of E2 in the development of lung cancer, which links hormone replacement therapy to increased lung cancer mortality (Gasperino, 2011). Protection measures against lung cancer deaths have also been observed in breast cancer survivors receiving endocrine therapy. It has been previously shown that the major ER homologous estrogen receptor $\beta-1$ (ER $\beta$ ) found in NSCLC is responsible for mediating the proliferation of estrogen, while the full-length $E R \alpha$ protein is usually not expressed. In preclinical models, estrogen blocking drugs have been studied for the treatment of lung cancer, and the way to show interaction with ER in lung cancer may be the common target.

In the study, changes of cellular endoplasmic reticulum and Golgi induced by the absence of CRABP2 expression occurred simultaneously with the expression of endoplasmic reticulum/Golgi stress regulatory protein. TEM results showed that ER silencing expanded endoplasmic reticulum and Golgi body structure. Therefore, the effects of endoplasmic reticulum/Golgi stress on en- doplasmic reticulum and Golgi Cytopainter were examined. The endoplasmic reticulum and Golgi were dyed red and green, respectively. In the control group, the endoplasmic reticulum tubules were initially localized in the perinuclear region of the control cells. While in the ER silencing cells, the cytoplasm related to endoplasmic reticulum was redistributed. The Golgi apparatus in the control group was strictly localized in the perinuclear region, and in the ER silencing cells the Golgi signal was redistributed in the cytoplasm. The expression levels of PERK, p-eIF2 $\alpha$, ATF4, and CHOP were analyzed by Western blotting assays. The expression levels of PERK, p-eIF2 alpha, ATF4 and CHOP in the control group were higher than those in the ER silencing group. The treatment of NSCLC has made great progress, but for most patients, the conventional treatment method cannot effectively lead to recurrence. Therefore, it is important to understand the occurrence and development of NSCLC, so as to promote treatment and improve the cure rate of patients.

In conclusion, the expression of CRABP2 in NSCLC cells was regulated by ER, and cell proliferation and invasion were regulated by the Hippo pathway. Meanwhile, the decrease of CRABP2 expression enhanced endoplasmic reticulum/Golgi stress response.

\section{REFERENCES}

Chen Q, Luo J, Wu C, Lu H, Cai S, Bao C, Liu D, Kong J (2020) The miRNA-149-5p/MyD88 axis is responsible for ursolic acidmediated attenuation of the stemness and chemoresistance of nonsmall cell lung cancer cells. Environ Toxicol 35: 561-569. https://doi. org/10.1002/tox. 22891

Cortesi E, Ventura J J (2019) Lgr6: From Stemness to Cancer Progression. J Lung Health Dis 3: 12-15

Feng X, Zhang M, Wang B, Zhou C, Mu Y, Li J, Liu X, Wang Y, Song Z, Liu P (2019) CRABP2 regulates invasion and metastasis of breast cancer through hippo pathway dependent on ER status. J Exp Clin Cancer Res 38: 361. https://doi.org/10.1186/s13046-019$1345-2$

Gasperino J (2011) Gender is a risk factor for lung cancer. Med Hypotheses 76: 328-331. https://doi.org/10.1016/j.mehy.2010.10.030

Gong F, Dong D, Zhang T, Xu W (2019) Long non-coding RNA FENDRR attenuates the stemness of non-small cell lung cancer cells via decreasing multidrug resistance gene 1 (MDR1) expression through competitively binding with RNA binding protein HuR. Eur J Pharmacol 853: 345-352. https://doi.org/10.1016/j. ejphar.2019.04.022

Guo L, Sun C, Xu S, Xu Y, Dong Q, Zhang L, Li W, Wang X, Ying G, Guo F (2019) Knockdown of long non-coding RNA linc-ITGB1 inhibits cancer stemness and epithelial-mesenchymal transition by reducing the expression of Snail in non-small cell lung cancer. Thorac Cancer 10: 128-136. https://doi.org/10.1111/17597714.12911

Han SS, Kim WJ, Hong Y, Hong SH, Lee SJ, Ryu DR, Lee W, Cho YH, Lee S, Ryu YJ, Won JY, Rhee H, Park JH, Jang SJ, Lee JS, Choi CM, Lee JC, Lee SD, Oh YM (2014) RNA sequencing identifies novel markers of non-small cell lung cancer. Lung Cancer 84: 229-235. https://doi.org/10.1016/j.lungcan.2014.03.018

Huang TH, Wu ATH, Cheng TS, Lin KT, Lai CJ, Hsieh HW, Chang PM, Wu CW, Huang CF, Chen KY (2019) In silico identification of thiostrepton as an inhibitor of cancer stem cell growth and an enhancer for chemotherapy in non-small-cell lung cancer. J Cell Mol Med 23: 8184-8195. https://doi.org/10.1111/jcmm.14689

Liu L, Yang Y, Liu S, Tao T, Cai J, Wu J, Guan H, Zhu X, He Z, Li J, Song E, Zeng M, Li M (2019) EGF-induced nuclear localization of SHCBP1 activates beta-catenin signaling and promotes cancer progression. Oncogene 38: 747-764. https://doi.org/10.1038/s41388018-0473-z

Masciale V, Grisendi G, Banchelli F, D'amico R, Maiorana A, Sighinolfi P, Stefani A, Morandi U, Dominici M,Aramini B (2019) Isolation and Identification of Cancer Stem-Like Cells in Adenocarcinoma and squamous cell carcinoma of the lung: A pilot study. Front Oncol 9: 1394. https://doi.org/10.3389/fonc.2019.01394

Miao F, Chen J, Shi M, Song Y, Chen Z, Pang L (2019) LncRNA HAND2-AS1 inhibits non-small cell lung cancer migration, invasion and maintains cell stemness through the interactions with TGF-beta1. Biosci Rep 39. https://doi.org/10.1042/BSR20181525 
Piao L, Yang Z, Feng Y, Zhang C, Cui C, Xuan Y (2019) LETM1 is a potential biomarker of prognosis in lung non-small cell carcinoma. BMC Cancer 19: 898. https://doi.org/10.1186/s12885-019-6128-9

Sung P J, Rama N, Imbach J, Fiore S, Ducarouge B, Neves D, Chen H W, Bernard D, Yang P C, Bernet A, Depil S, Mehlen P (2019) Cancer-associated fibroblasts produce netrin-1 to control cancer cell plasticity. Cancer Res 79: 3651-3661. https://doi.org/10.1158/00085472.CAN-18-2952

Ware KE, Hinz TK, Kleczko E, Singleton KR, Marek LA, Helfrich BA, Cummings CT, Graham DK, Astling D, Tan AC, Heasley LE (2013) A mechanism of resistance to gefitinib mediated by cellular reprogramming and the acquisition of an FGF2-FGFR1 autocrine growth loop. Oncogenesis 2: e39. https://doi.org/10.1038/oncsis. 2013.4

Wei L, Liu Y, Ma Y, Ding C, Zhang H, Lu Z, Gu Z, Zhu C (2019) C-X-C chemokine receptor 2 correlates with unfavorable prognosis and facilitates malignant cell activities via activating JAK2/STAT3 pathway in non-small cell lung cancer. Cell Cycle 18: 3456-3471. https://doi.org/10.1080/15384101.2019.1689471

Weiss J, Sos ML, Seidel D, Peifer M, Zander T, Heuckmann JM, Ullrich RT, Menon R, Maier S, Soltermann A, Moch H, Wagener P, Fischer F, Heynck S, Koker M, Schottle J, Leenders F, Gabler F, Dabow I, Querings S, Heukamp LC, Balke-Want H, Ansen S, Rauh D, Baessmann I, Altmuller J, Wainer Z, Conron M, Wright G, Russell P, Solomon B, Brambilla E, Brambilla C, Lorimier P, Sollberg S, Brustugun O T, Engel-Riedel W, Ludwig C, Petersen I, Sanger J, Clement J, Groen H, Timens W, Sietsma H, Thunnissen E, Smit E, Heideman D, Cappuzzo F, Ligorio C, Damiani S, Hallek M, Beroukhim R, Pao W, Klebl B, Baumann M, Buettner R, Ernestus K, Stoelben E, Wolf J, Nurnberg P, Perner S, Thomas RK (2010) Frequent and focal FGFR1 amplification associates with therapeutically tractable FGFR1 dependency in squamous cell lung cancer. $S_{c i}$ Transl Med 2: 62ra93. https://doi.org/10.1126/scitranslmed.3001451

Weng CH, Chen LY, Lin YC, Shih JY, Lin YC, Tseng RY, Chiu AC, Yeh YH, Liu C, Lin YT, Fang JM, Chen CC (2019) Epithelialmesenchymal transition (EMT) beyond EGFR mutations per se is a common mechanism for acquired resistance to EGFR TKI. Oncogene 38: 455-468. https://doi.org/10.1038/s41388-018-0454-2
Wu JI, Lin YP, Tseng CW, Chen HJ, Wang LH (2019) Crabp2 promotes metastasis of lung cancer cells via HuR and integrin beta1/ FAK/ERK signaling. Sci Rep 9: 845. https://doi.org/10.1038/ s41598-018-37443-4

Xia D, Chen B, Yang X (2019) Correlation of integrin alpha 7 with clinicopathological characteristics and survival profiles, as well as its regulatory role in cell proliferation, apoptosis, and stemness in non-small-cell lung cancer. J Clin Lab Anal 33: e22973. https://doi. org/10.1002/jcla.22973

Yang L, Dong Y, Li Y, Wang D, Liu S, Wang D, Gao Q, Ji S, Chen X, Lei Q, Jiang W, Wang L, Zhang B, Yu JJ, Zhang Y (2019) IL-10 derived from M2 macrophage promotes cancer stemness via JAK1/ STAT1/NF-kappaB/Notch1 pathway in non-small cell lung cancer. Int J Cancer 145: 1099-1110. https://doi.org/10.1002/ijc.32151

Zhang F, Wang J, Wang X, Wei N, Liu H, Zhang X (2019a) CD146mediated acquisition of stemness phenotype enhances tumour invasion and metastasis after EGFR-TKI resistance in lung cancer. Clin Respir J 13: 23-33. https://doi.org/10.1111/cri.12976

Zhang L, Yang Y, Chai L, Bu H, Yang Y, Huang H, Ran J, Zhu Y, Li L, Chen F, Li W (2020) FRK plays an oncogenic role in non-small cell lung cancer by enhancing the stemness phenotype via induction of metabolic reprogramming. Int J Cancer 146: 208-222. https://doi. org/10.1002/ijc.32530

Zhang X, Yang B, Shao D, Zhao Y, Sun J, Li J, Li Y, Cao F (2019b) Longitudinal association of subjective prospective and retrospective memory and depression among patients with glioma. Eur J Oncol Nurs 42: 1-6. https://doi.org/10.1016/j.ejon.2019.07.003

Zhao G, Zhang Y, Zhao Z, Cai H, Zhao X, Yang T, Chen W, Yao C, Wang Z, Wang Z, Han C, Wang H (2020) MiR-153 reduces stem cell-like phenotype and tumor growth of lung adenocarcinoma by targeting Jagged1. Stem Cell Res Ther 11: 170. https://doi. org/10.1186/s13287-020-01679-7

Zhu L, Pan R, Zhou D, Ye G, Tan W (2019) BCL11A enhances stemness and promotes progression by activating Wnt/beta-catenin signaling in breast cancer. Cancer Manag Res 11: 2997-3007. https:// doi.org/10.2147/CMAR.S199368 